\title{
Relaciones alométricas para predecir biomasa en plantas de Pinus pseudostrobus cultivadas en diferentes sistemas de producción en vivero
}

\author{
Allometric relations for predicting biomass in Pinus pseudostrobus seedlings grown \\ under different production systems at nursery
}

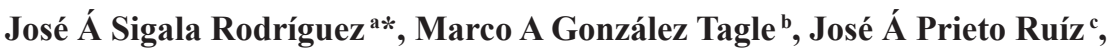 \\ Erickson Basave Villalobos ${ }^{a}$, Javier Jiménez Pérez ${ }^{\mathrm{b}}$
}

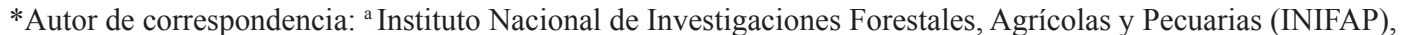 \\ Campo Experimental Valle del Guadiana, Km 4.5 Carretera Durango-El Mezquital C.P. 34170 Durango, Durango, México, \\ tel.: +52 (55) 38718700, sigala.jose@inifap.gob.mx \\ ${ }^{\mathrm{b}}$ Universidad Autónoma de Nuevo León, Facultad de Ciencias Forestales, Linares, Nuevo León, México.

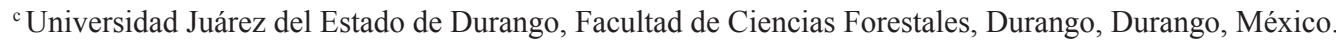

\begin{abstract}
SUMMARY
The analysis of nursery seedling quality requires measures of content and distribution of seedling biomass, which involves constant destructive sampling. To avoid this, it is possible to get biomass estimations throughout allometric equations from easy-measure variables; however, it is necessary to compute these relationships by species and for the different production environments. The aim of the study was to identify allometric relations between biomass and variables as root collar diameter (D), shoot height $(\mathrm{H})$ and $\mathrm{D}^{2} \mathrm{H}$ of Pinus pseudostrobus seedlings growing under different production systems, with the purpose of defining if there is an allometric equation for each production system which could estimate total, aboveground and root biomass of seedlings. The analysis of covariance was performed to evaluate the effect of the production system on the relation between biomass and independent variables $\left(\mathrm{D}, \mathrm{H}, \mathrm{D}^{2} \mathrm{H}\right)$. Influence of the production system on intercepts and slopes of some relations between biomass was found. The full models to predict aboveground biomass and total biomass, considering the production system, had better goodness-fit statistics than those belonging to reduced models; whereas for root biomass, the reduced models shown less relative prediction error than those shown by full models. Root collar diameter explains most biomass variation for the three components
\end{abstract}

Key words: diameter, seedling, pine, model, allometry.

\section{RESUMEN}

El análisis de calidad de planta forestal en vivero requiere la medición del contenido y distribución de biomasa en las plantas, lo que implica constantes muestreos destructivos. Para evitar esto, es posible estimar la biomasa mediante ecuaciones alométricas a partir de variables predictivas de fácil medición; sin embargo, es necesario determinar estas relaciones por especie y para los diferentes ambientes de producción. El objetivo del estudio fue identificar relaciones alométricas entre la biomasa y las variables diámetro al cuello de la raíz (D), altura del tallo (H) y D² $\mathrm{H}$ en plantas de Pinus pseudostrobus cultivadas bajo diferentes sistemas de producción en vivero, con la finalidad de determinar si existe una ecuación alométrica para cada sistema de producción con la que se estime la biomasa total de la planta y de sus componentes. Se realizó un análisis de covarianza para probar el efecto del sistema de producción sobre las relaciones alométricas de biomasa y las variables $\mathrm{D}, \mathrm{H}$ o $\mathrm{D}^{2} \mathrm{H}$. Se encontró efecto del sistema de producción sobre el intercepto y pendiente de algunas relaciones. Los modelos completos para biomasa aérea y total, tomando en cuenta el efecto del sistema de producción, presentaron mejor ajuste y precisión que los modelos reducidos sin considerar el sistema de producción, mientras que para la biomasa de raíz, los modelos reducidos mostraron menor error relativo de predicción. Para los tres componentes de biomasa, el diámetro fue la variable que explicó en mayor proporción la variación en biomasa.

Palabras clave: diámetro, planta, pino, modelo, alometría.

\section{INTRODUCCIÓN}

La biomasa se emplea como una variable para analizar la calidad de planta forestal como respuesta de la planta al ambiente y a diferentes prácticas de cultivo en vivero (Bloomberg et al. 2008). En México, la Comisión Nacio- nal Forestal busca reglamentar los indicadores de calidad de planta estableciendo estándares en variables morfológicas, como la altura del tallo y el diámetro al cuello de la raíz, por su facilidad de medición (CONAFOR 2011). Por otra parte, existen indicadores como la relación biomasa tallo/raíz o bien el índice de calidad de Dickson et al. 
(1960) que se han definido como criterios apropiados para predecir desempeño de las plantas en campo (Grossnickle 2012, Tsakaldimi et al. 2013); no obstante, el cálculo de estos índices requiere la estimación de la biomasa aérea, de raíz y total en las plantas, mediante muestreos destructivos constantes en cada ciclo de producción (Stape et al. 2001).

La principal desventaja del muestreo destructivo es que no puede ser utilizado para mediciones repetidas en un solo individuo a lo largo de un periodo de tiempo (Bloomberg et al. 2008). Una alternativa para estimar la biomasa sin muestreos destructivos, es el uso de relaciones alométricas con las mismas variables morfológicas comúnmente analizadas en las plantas, como el diámetro al cuello de la raíz (D) y altura de la planta $(\mathrm{H})$ o algunas variables derivadas $\left(\mathrm{D}^{2}\right.$ o $\left.\mathrm{D}^{2} \mathrm{H}\right)$ (Ruehle et al. 1984, Geudens et al. 2004, Bloomberg et al. 2008). Una ecuación alométrica describe una línea curva que representa la relación entre la biomasa y el diámetro o la altura de la planta (Schmidt et al. 2009); además, proporciona un buen equilibrio y precisión en las predicciones con bajos requerimientos de datos (Ter-Mikaelian y Korzukhin 1997).

En México, Pinus pseudostrobus Lindl. es una de las especies más propagadas para atender programas de reforestación, con una producción anual cercana a 19 millones de plantas, cultivadas tanto en sistema tradicional (bolsa de polietileno) como en tecnificado (bandejas de poliestireno) (CONAFOR 2011). Para esta especie se han desarrollado tablas de rendimiento en biomasa con base en funciones alométricas en árboles de poblaciones naturales y/o plantaciones (Domínguez et al. 2009, Aguirre y Jiménez 2011, Méndez et al. 2011); sin embargo, los umbrales mínimos de las tablas de rendimiento no permiten la estimación de biomasa en plantas o árboles jóvenes (Blujdea et al. 2011), y si se calculara, la proyección llevaría a grandes errores, debido principalmente a las diferencias en la densidad de tejidos leñosos entre plantas y árboles adultos (Preece et al. 2015).

Por ello, el objetivo del presente estudio es identificar relaciones alométricas en plantas de Pinus pseudostrobus, específicas para los sistemas de producción empleados por los viveristas para estimar biomasa de los diferentes componentes (parte aérea, raíz y total); la hipótesis planteada es que una ecuación alométrica específica para plantas en condiciones de vivero puede ser utilizada en la estimación de biomasa con buen grado de ajuste y aplicación para el monitoreo de los distintos índices de calidad de planta en diferentes sistemas de producción.

\section{MÉTODOS}

Material vegetal. Se utilizaron plantas de Pinus pseudostrobus producidas en el vivero forestal del Bosque Escuela de la Facultad de Ciencias Forestales (UANL), ubicado en el Ejido Santa Rosa, municipio de Iturbide, Nuevo León, en las coordenadas $24^{\circ} 42,37^{\prime} \mathrm{N}$ y $99^{\circ} 51,69^{\prime} \mathrm{O}$ y altitud de $1.608 \mathrm{~m}$. Se trabajó con lotes provenientes de tres sistemas de producción (cuadro 1).

En los tres sistemas, el sustrato estuvo compuesto por $57 \%$ de turba de musgo (peat moss) y $43 \%$ de tierra común, adicionado con $5 \mathrm{~kg} \mathrm{~m}^{-3}$ de fertilizante de liberación controlada (Osmocote $\AA$, 18-6-12 NPK). Las plantas se cultivaron un año bajo condiciones de malla sombra y, en el caso del sistema en bolsa de polietileno, en el segundo año se mantuvieron a la intemperie. Durante el periodo de producción se aplicaron riegos cada tres días de acuerdo con las rutinas convencionales del vivero.

Desarrollo de ecuaciones. En septiembre de 2011 se realizó un muestreo destructivo al azar a una intensidad del $0,15 \%$ de cada lote de plantas. En el sistema de bandeja de poliestireno, el muestreo consistió en elegir 25 bandejas al azar de todo el lote y de cada bandeja se extrajeron tres plantas al azar; en el caso de las plantas en bolsa, que estuvieron acomodadas en seis platabandas de 5.000 plantas, se extrajeron al azar ocho plantas de cada platabanda.

De las plantas muestreadas se midió el diámetro al cuello de la raíz $(\mathrm{D}, \mathrm{mm})$, altura del tallo $(\mathrm{H}, \mathrm{cm})$ y biomasa $(\mathrm{B}, \mathrm{g})$; esta última fue calculada mediante la medición del peso seco del material vegetal, que se deshidrató en estufa de secado a $60{ }^{\circ} \mathrm{C}$ durante 72 horas. Esta variable fue dividida en biomasa de la parte aérea $\left(B_{A}\right)$, biomasa de la raíz $\left(B_{R}\right)$ y biomasa total $\left(B_{T}\right)$.

Para el desarrollo de las ecuaciones, se partió del modelo general para estimar la biomasa total y de los componentes de las plantas:

$$
B=\beta_{0} X^{\beta_{1}}
$$

Cuadro 1. Sistemas de producción para plantas de Pinus pseudostrobus en el vivero del Bosque Escuela (FCF-UANL).

Production systems for Pinus pseudostrobus seedlings at Bosque Escuela nursery (UANL).

\begin{tabular}{lcccc}
\hline Sistema de producción & Siembra & Volumen de contenedor & Edad* (años) & Población \\
\hline Bandeja de poliestireno de 160 cavidades & Septiembre 2010 & $60 \mathrm{~cm}^{3}$ & $1+0$ & 50.000 \\
Bolsa de polietileno & Septiembre 2009 & $630 \mathrm{~cm}^{3}$ & $0+2$ & 21.330 \\
Bandeja de poliestireno - Bolsa de polietileno & Septiembre 2009 & $60-560 \mathrm{~cm}^{3}$ & $1+1$ & 10.660 \\
\hline
\end{tabular}

* El primer número indica cultivo en bandeja y el segundo cultivo en bolsa. 
donde: $B=$ biomasa seca y $X=$ diámetro $(\mathrm{D})$, altura $(\mathrm{H})$ o $\mathrm{D}^{2} \mathrm{H} ; \beta_{0}$ y $\beta_{1}=$ parámetros (intercepto y pendiente) del modelo (Ter-Mikaelian y Parker 2000, Bloomberg et al. 2008, Schmidt et al. 2009).

Los parámetros fueron estimados por el procedimiento de mínimos cuadrados convirtiendo la ecuación 1 a su forma lineal mediante la transformación logarítmica de las variables (Ter-Mikaelian y Parker 2000):

$$
\ln B=\ln \beta_{0}+\beta_{1} \ln X
$$

La ecuación 2 representó un modelo reducido que describe todos los datos sin considerar el sistema de producción. Para incluir este factor, la ecuación 2 fue especificada como un modelo completo de análisis de covarianza (ANCOVA) con parámetros $\beta_{0}$ y $\beta_{l}$ diferentes en cada sistema de producción, en el cual $\mathrm{D}, \mathrm{H}$ o $\mathrm{D}^{2} \mathrm{H}$ fueron variables continuas independientes o covariables y el sistema de producción fue el efecto fijo (Bloomberg et al. 2008):

$$
\ln B_{i j}=\left(\mu+\alpha_{i}\right)+\left(\beta_{j}+\gamma_{i}\right) \ln X_{i j}
$$

donde:

$B_{i j}=$ valor esperado de biomasa para una planta,

$X_{i j}^{i j}=$ valor de la variable de la misma planta,

$\mu=$ intercepto y $\beta$ es la pendiente de la relación biomasa y la variable,

$\alpha_{i}$ y $\gamma_{i}=$ diferencias en intercepto y pendiente entre los sistemas de producción (bandeja $[i=1]$, bolsa $[i=2]$ y bandeja-bolsa $[i=3]$ ); por lo tanto, en la ecuación $2, \beta_{0}=$ $\left(\mu+\alpha_{i}\right)$ y $\beta_{1}=\left(\beta+\gamma_{i}\right)$

Ambos modelos (ecuaciones 2 y 3 ) se reprodujeron para los tres componentes de biomasa (parte aérea, raíz y total) a partir del grupo de datos obtenidos del primer muestreo. Se utilizó el procedimiento GLM de SAS versión 9.2 (SAS 2009) y la prueba de $\mathrm{t}(\alpha<0,05)$ para probar la significancia estadística de los parámetros en cada modelo.

Validación de ecuaciones. Después del muestreo, 180 plantas por cada sistema de producción fueron aleatoriamente seleccionadas y separadas para mantenerlas creciendo bajo las mismas condiciones durante 45 días y en ellas validar las ecuaciones generadas. De estos lotes compactos, una muestra destructiva de 36 plantas al azar fue tomada para evaluar nuevamente las variables morfológicas.

Asimismo, la biomasa del grupo de datos de validación fue calculada mediante las ecuaciones generadas para evaluar el grado de ajuste de los modelos. Debido a que los modelos fueron desarrollados en unidades logarítmicas, al transformar la ecuación a su forma original (ecuación 1) se sumó la mitad del error estándar al cuadrado $\left(1 / 2(S x y)^{2}\right)$ del intercepto $\ln \left(\beta_{0}\right)$, para corregir el sesgo introducido por la transformación logarítmica (Sprugel 1983). El ajuste de los datos de validación fue probado usando el error relativo de predicción (ERP-\%), calculado como la diferencia entre el valor observado y predicho, dividido por el valor observado (Ter-Mikaelian y Parker 2000); con ello el valor de biomasa predicho por el modelo fue comparado con el valor observado en los datos de validación.

\section{RESULTADOS}

En el grupo de datos de calibración, los valores de diámetro fluctuaron de 2,7 a $5,8 \mathrm{~mm}$ y la altura de 8,5 a 25,9 $\mathrm{cm}$, mientras que la biomasa total estimada fue de 0,8 a 6,1 $\mathrm{g}$ por planta. En los datos de validación, las plantas presentaron valores de 2,8 a $5,7 \mathrm{~mm}$ de diámetro, 9,3 a $25,2 \mathrm{~cm}$ de altura y 0,8 a $5,7 \mathrm{~g}$ de biomasa por planta (cuadro 2).

Cuadro 2. Media y error estándar de las variables morfológicas usadas en el conjunto de datos de calibración y validación para el desarrollo de las ecuaciones.

Descriptive statistics which summarizes both calibration and validation datasets used to develop the equations.

\begin{tabular}{lccccc}
\hline & Diámetro $(\mathrm{mm})$ & Altura $(\mathrm{cm})$ & \multicolumn{3}{c}{ Biomasa $(\mathrm{g})$} \\
\cline { 5 - 6 } & & & Parte aérea & Raíz & Total \\
\hline Datos de calibración $(\mathrm{n}=123)$ & & & & & \\
\hline Bandeja $(1+0)$ & $2,71 \pm 0,14$ & $8,53 \pm 0,38$ & $0,52 \pm 0,06$ & $0,25 \pm 0,03$ & $0,77 \pm 0,08$ \\
Bolsa (0+2) & $5,79 \pm 0,37$ & $25,5 \pm 1,95$ & $4,75 \pm 0,78$ & $1,38 \pm 0,17$ & $6,14 \pm 0,92$ \\
Bandeja-Bolsa (1+1) & $5,37 \pm 0,61$ & $14,9 \pm 1,73$ & $2,64 \pm 0,62$ & $0,92 \pm 0,17$ & $3,56 \pm 0,76$ \\
\hline Datos de validación $(\mathrm{n}=108)$ & & & & & \\
\hline Bandeja (1+0) & $2,84 \pm 0,15$ & $9,33 \pm 0,59$ & $0,57 \pm 0,08$ & $0,28 \pm 0,05$ & $0,85 \pm 0,12$ \\
Bolsa (0+2) & $5,74 \pm 0,39$ & $25,2 \pm 1,74$ & $4,09 \pm 0,58$ & $1,61 \pm 0,19$ & $5,69 \pm 0,74$ \\
Bandeja-Bolsa $(1+1)$ & $5,69 \pm 0,33$ & $15,4 \pm 1,35$ & $2,87 \pm 0,32$ & $1,44 \pm 0,18$ & $4,31 \pm 0,48$ \\
\hline
\end{tabular}


Calibración de ecuaciones. El modelo reducido y ajustado a la forma lineal (ecuación 2) presentó alta significancia estadística en todas las relaciones alométricas $(P<0,0001)$. Todas las ecuaciones presentaron valores de $\mathrm{R}^{2}>0,90$, con mejor ajuste y menor error estándar en las ecuaciones donde se incluyeron las dos variables independientes, diámetro (D) y altura (H), aunque el diámetro como variable independiente explicó la mayor parte de la variación en la biomasa (cuadro 3). En los tres modelos probados (D, D² H y $[\mathrm{D}, \mathrm{H}]$ ) se obtuvo menor ajuste en las ecuaciones para calcular biomasa de la raíz $\left(B_{R}\right)$ con valores de $\mathrm{R}^{2}$ inferiores o igual a 0,91 ; en cambio, los modelos para la biomasa de la parte aérea $\left(B_{A}\right)$ y biomasa total $\left(B_{T}\right)$ mostraron mejor ajuste, con valores de $\mathrm{R}^{2}$ entre 0,94 y 0,97 .

En el modelo completo (ecuación 3), los parámetros y de algunas de las relaciones alométricas, difirieron estadísticamente entre sistemas de producción, principalmente para las variables $B_{A}$ y $B_{T}$. En la figura 1 se presenta la relación entre la biomasa y las variables morfológicas explicativas después de la transformación logarítmica $\left(\operatorname{lnD}\right.$ y $\left.\ln \mathrm{D}^{2} \mathrm{H}\right)$, observándose una tendencia diferente para cada sistema de producción.

Al usar D como variable independiente en el modelo completo, el sistema de producción no tuvo efecto significativo sobre el intercepto y la pendiente; no obstante, al usar la variable independiente $\mathrm{D}^{2} \mathrm{H}$, el intercepto de la relación para biomasa total $\left(B_{T}\right)$ fue estadísticamente diferente $(P=0,005)$ entre el sistema de producción bandeja-bolsa $(1+1)$ y el sistema bandeja $(1+0)$. Este efecto también fue significativo para la biomasa de la parte aérea $\left(B_{A}\right)$; pero no para la biomasa de la raíz $\left(B_{R}\right)$ pues no se encontraron diferencias del intercepto y pendiente de la relación alométrica en función del sistema de producción.

Por otra parte, al usar D y $\mathrm{H}$ como variables independientes en relación con $B_{T}$ y $B_{A}$, se encontró un efecto significativo del sistema bandeja-bolsa (1+1) tanto en el intercepto $(P<0,01)$ como en la pendiente del modelo $(P<0,001)$; en cambio, para $B_{R}$ el intercepto y pendiente de la relación no fueron diferentes entre sistemas de producción (cuadro 4).

El estimador de la pendiente en el sistema de producción bandeja-bolsa $(1+1)$ fue negativo para la relación $\mathrm{H}$ y los tres componentes de biomasa, explicado por un menor crecimiento en altura de las plantas que fueron trasplantadas de bandeja a bolsa. En la figura 2 se muestra que la relación entre biomasa total $\left(B_{T}\right)$ y D tiene una tendencia similar en cada sistema producción, así como para el modelo reducido; en cambio, cuando se incluye la variable altura de la forma $\mathrm{D}^{2} \mathrm{H}$, esta relación difiere para los tres sistemas de producción.

Validación de ecuaciones. Los promedios del error relativo de predicción (ERP) para los modelos usando los datos de validación, muestran la superioridad de los modelos completos. Este error fue menor para la estimación de $B_{T}$, con valores que fluctuaron de 2,30 a 7,66 \%; el menor valor se obtuvo al utilizar D como variable predictiva en el modelo completo. Para la estimación de $B_{A}$, el menor ERP $(9,49 \%)$ se obtuvo con el modelo completo incluyendo $\mathrm{D}^{2} \mathrm{H}$ variable predictiva (cuadro 5). Por otra parte, el error relativo de predicción para estimar biomasa de la raíz fue negativo tanto en el modelo reducido como en el modelo completo, con valores de $-6,78$ a $-9,45 \%$, observándose menor error de predicción con el modelo reducido, principalmente usando D como variable explicativa (cuadro 5).

En la figura 3, que describe la biomasa total predicha contra la biomasa total observada para los datos de validación, se muestra que las predicciones del modelo completo usando D como variable independiente resultó ser más preciso que el modelo reducido, ya que este último subestima la biomasa en el sistema bandeja-bolsa $(1+1)$ y la sobrestima para el sistema de bolsa $(0+2)$. Por otra parte, cuando se incluye la variable altura $\left(\mathrm{D}^{2} \mathrm{H}\right.$ o $\left.\mathrm{D}, \mathrm{H}\right)$, disminuye la diferencia en la precisión de la predicción entre el modelo completo y el reducido.

Cuadro 3. Parámetros del modelo reducido ajustado a la forma lineal después de la transformación logarítmica $\left(\ln B=\ln \beta_{0}+\beta_{1} \ln X\right)$. Reduced model parameters adjusted to a linear form after logarithmic transformation $\left(\ln B=\ln \beta_{0}+\beta_{1} \ln X\right)$.

\begin{tabular}{cccccccc}
\hline $\mathrm{Y}$ & $\mathrm{X}$ & $\beta_{0}\left(\mathrm{Sxy}^{*}\right)$ & $\beta_{1}$ & $\beta_{2}$ & $\mathrm{R}^{2}$ & $\mathrm{Sxy}^{*}$ & $P>\mathrm{F}$ \\
\hline$B_{A}$ & $\mathrm{D}$ & $-3,176(0,079)$ & $2,547(0,059)$ & --- & 0,94 & 0,272 & $<0,0001$ \\
$B_{A}$ & $\mathrm{D}^{2} \mathrm{H}$ & $-0290(0,019)$ & $0,824(0,014)$ & --- & 0,96 & 0,203 & $<0,0001$ \\
$B_{A}$ & $\mathrm{D}, \mathrm{H}$ & $-3,958(0,097)$ & $1,803(0,086)$ & $0,694(0,069)$ & 0,97 & 0,201 & $<0,0001$ \\
$B_{R}$ & $\mathrm{D}$ & $-3,477(0,083)$ & $2,07(0,062)$ & --- & 0,90 & 0,285 & $<0,0001$ \\
$B_{R}$ & $\mathrm{D} 2 \mathrm{H}$ & $-1,127(0,027)$ & $0,661(0,02)$ & --- & 0,90 & 0,282 & $<0,0001$ \\
$B_{R}$ & $\mathrm{D}, \mathrm{H}$ & $-3,877(0,131)$ & $1,69(0,116)$ & $0,355(0,093)$ & 0,91 & 0,270 & $<0,0001$ \\
$B_{T}$ & $\mathrm{D}$ & $-2,651(0,072)$ & $2,41(0,054)$ & --- & 0,94 & 0,249 & $<0,0001$ \\
$B_{T}$ & $\mathrm{D}^{2} \mathrm{H}$ & $0,081(0,019)$ & $0,777(0,014)$ & --- & 0,96 & 0,198 & $<0,0001$ \\
$B_{T}$ & $\mathrm{D}, \mathrm{H}$ & $-3,326(0,093)$ & $1,769(0,083)$ & $0,598(0,066)$ & 0,96 & 0,193 & $<0,0001$ \\
\hline
\end{tabular}

*Sxy = error estándar en unidades logarítmicas.

$* \mathrm{Sxy}=$ standard error in logarithmic units. 


\section{DISCUSIÓN}

De acuerdo con los resultados obtenidos, existe una ecuación alométrica con buenos estadísticos de ajuste, que estima la biomasa total y aérea en plantas de Pinus pseudostrobus a partir de la altura y el diámetro al cuello de la raíz como variables predictivas, que distingue el sistema de producción. Al respecto, Bloomberg et al. (2008) y Jackson et al. (2012) concuerdan en que las relaciones
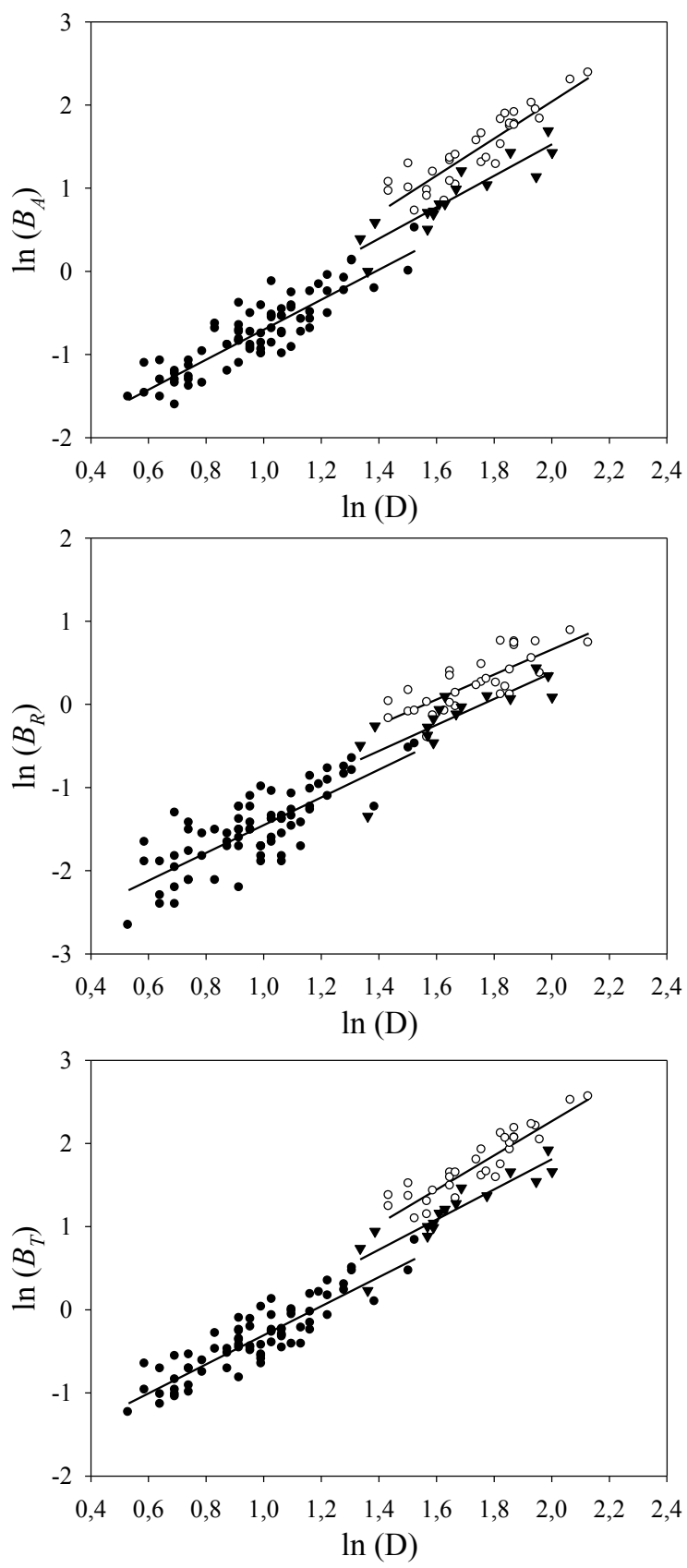

alométricas en plantas cultivadas en vivero son afectadas por el sistema de producción y las condiciones de cultivo. Igualmente, otros autores señalan que distintos factores pueden influir en las relaciones alométricas de las plantas, como la edad (Geudens et al. 2004), el estrés debido al trasplante (Ortega et al. 2006), la densidad de cultivo y el volumen del contenedor (Del Campo et al. 2011); incluso, pueden diferir entre especies de coníferas (Takahashi y Obata 2014).
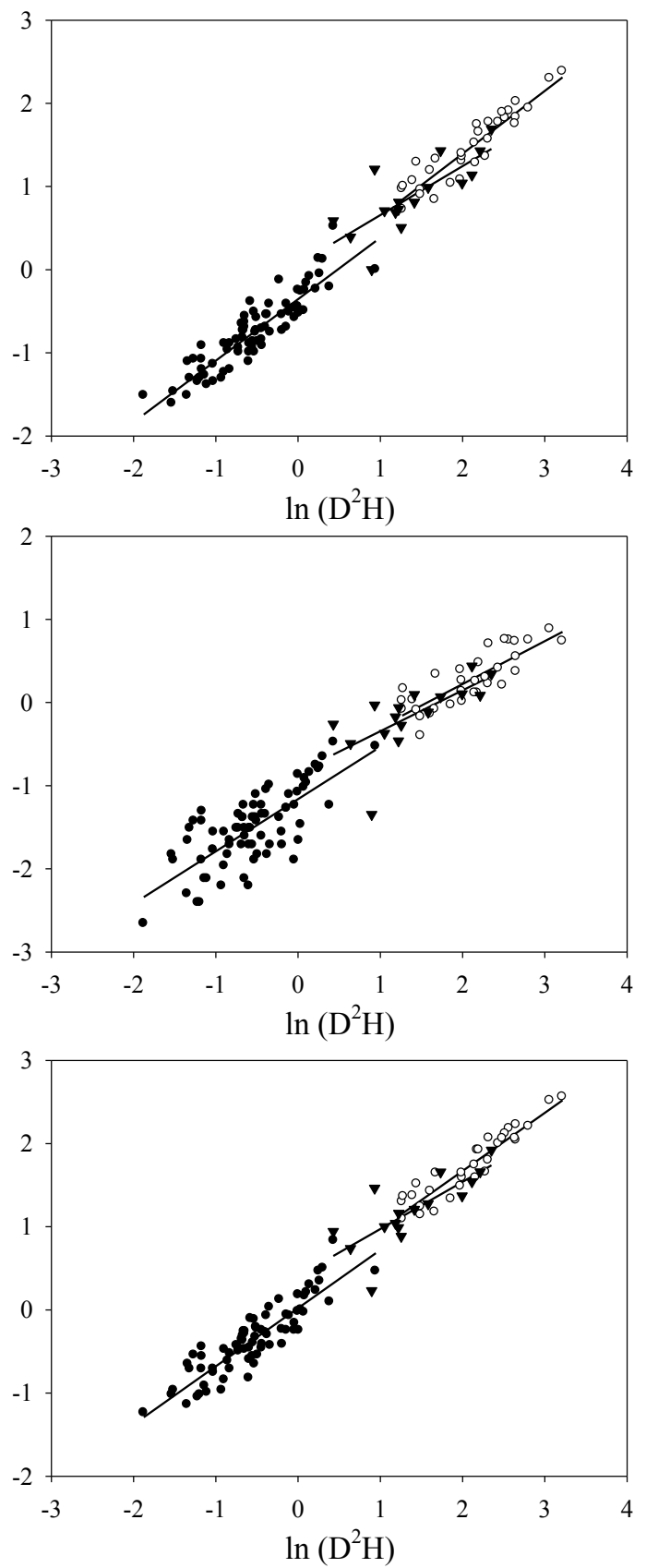

Figura 1. Dispersión de datos de biomasa $\left(\ln B_{A}, \ln B_{R}\right.$ y $\left.\ln B_{T}\right)$ contra las variables independientes $\left(\operatorname{lnD}\right.$ y $\left.\ln \mathrm{D}^{2} \mathrm{H}\right)$ y líneas de tendencia en la forma para cada sistema de producción.

Dispersion of biomass datasets $\left(\ln B_{A}, \ln B_{R}\right.$ and $\left.\ln B_{T}\right)$ vs. independent variables $\left(\ln \mathrm{D}\right.$ and $\left.\ln \mathrm{n}^{2} \mathrm{H}\right)$ and tendency line fitted to , for each production system. 


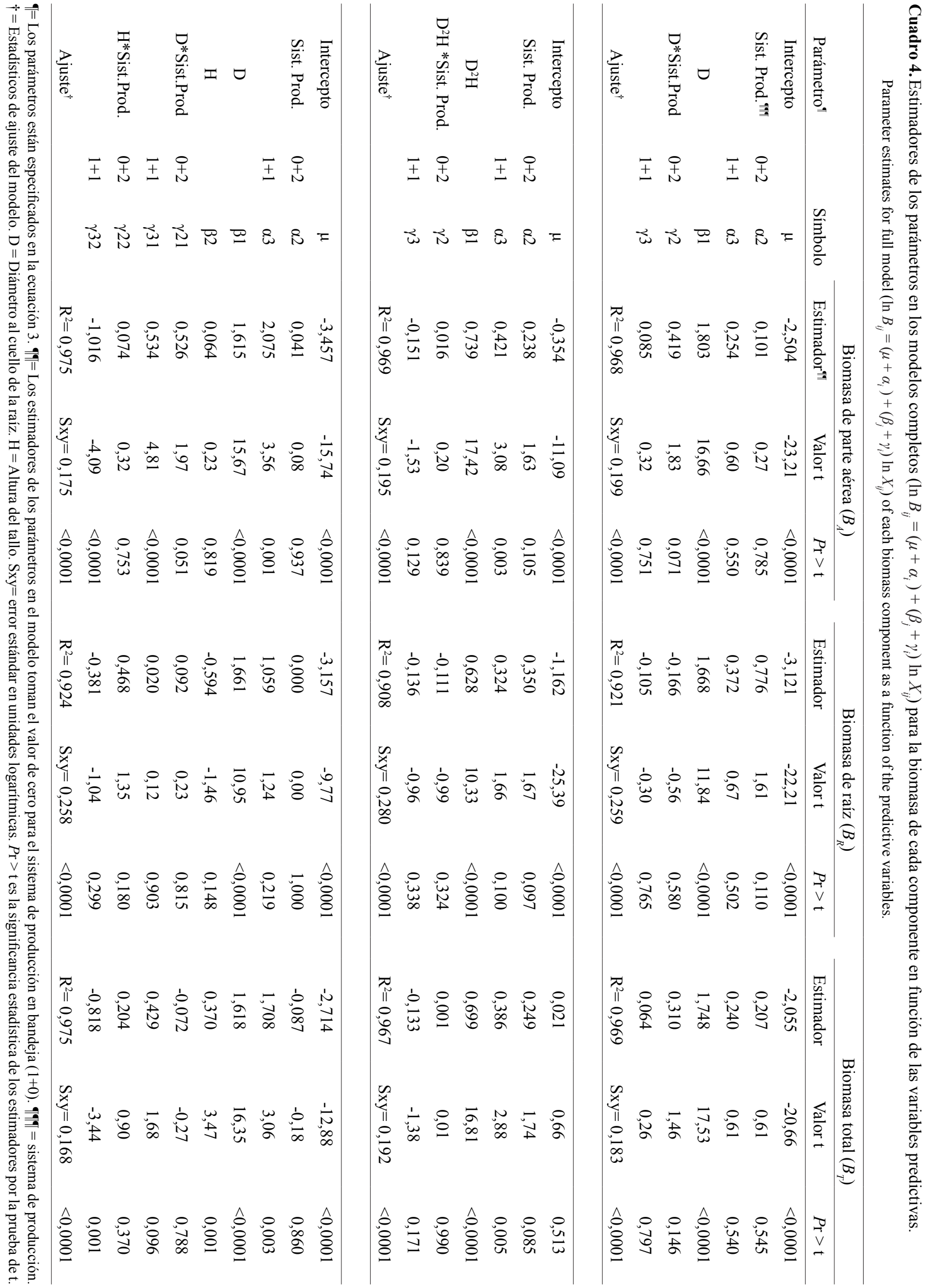


El diámetro fue la variable que explicó en mayor proporción la biomasa de las plantas, ya que valor de $\mathrm{R}^{2}$ del modelo completo al estimar biomasa total fue de 0,969 ( $P$ $<0,0001)$ en la ecuación que solo considera el diámetro como variable independiente, y este se incrementó sólo a $0,975(P<0,0001)$ agregando la altura; sin embargo, el menor error relativo de predicción $(2,3 \%)$ fue obtenido utilizando únicamente D en el modelo completo. En este sentido, Ter-Mikaelian y Parker (2000), Miguel et al. (2010) y Blujdea et al. (2011) coinciden en que el diámetro es el parámetro que más contribuye en una ecuación alométrica usada para estimar biomasa en plántulas; asimismo, Chaturvedi y Raghubanshi (2013) sugieren que es más práctico utilizar modelos incluyendo sólo el diámetro como variable independiente, siempre y cuando las ganancias en precisión del modelo incluyendo la altura, no sean biológicamente relevantes.

En contraparte, Lödige et al. (2014) mencionan que el patrón de distribución de la biomasa aérea puede estar controlado principalmente por la altura de la planta; este comportamiento, pudo ser apreciado en los modelos reducidos, en los cuales, al incluir la altura en la forma $\mathrm{D}^{2} \mathrm{H}$ o [D, H], se disminuyó sustancialmente el error relativo de predicción. De la misma forma, el menor error relativo de predicción $(9,49 \%)$, para el cálculo de biomasa aérea, fue obtenido con la variable $\mathrm{D}^{2} \mathrm{H}$ en el modelo completo.

Tanto para el modelo reducido como para el completo, los valores de $\mathrm{R}^{2}$ en la estimación de biomasa aérea y to-
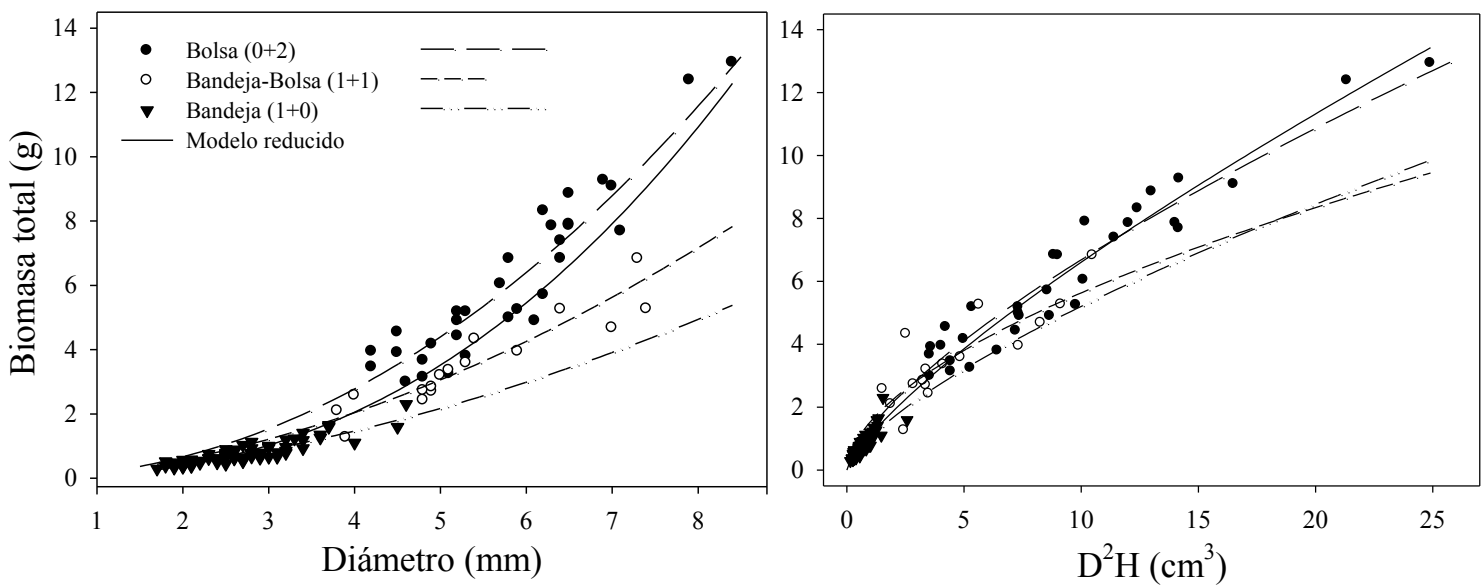

Figura 2. Biomasa total en función de $\mathrm{D}$ o $\mathrm{D}^{2} \mathrm{H}$ : modelo completo para los diferentes sistemas de producción, así como el modelo reducido sin considerar el sistema de producción. duction system.

Total biomass as a function of $\mathrm{D}$ o $\mathrm{D}^{2} \mathrm{H}$ : Full model for the different production systems and reduced model without considering the pro-

Cuadro 5. Media y desviación estándar del error relativo de predicción para el conjunto de datos de validación.

Mean and standard deviation of relative prediction error (RPE) for validation dataset.

\begin{tabular}{|c|c|c|c|c|c|c|}
\hline \multirow{2}{*}{ Modelo } & \multicolumn{2}{|c|}{ Biomasa de la parte aérea $\left(B_{A}\right)$} & \multicolumn{2}{|c|}{ Biomasa de la raíz $\left(B_{R}\right)$} & \multicolumn{2}{|c|}{ Biomasa total $\left(B_{T}\right)$} \\
\hline & Media (\%) & SD & Media (\%) & SD & Media (\%) & SD \\
\hline \multicolumn{7}{|l|}{ Modelo reducido } \\
\hline $\mathrm{D}$ & 16,38 & 34,17 & $-6,78$ & 28,99 & 7,66 & 27,79 \\
\hline $\mathrm{D}^{2} \mathrm{H}$ & 10,29 & 23,97 & $-8,56$ & 29,56 & 3,19 & 21,66 \\
\hline $\mathrm{D}, \mathrm{H}$ & 10,86 & 23,61 & $-8,09$ & 27,64 & 3,80 & 20,73 \\
\hline \multicolumn{7}{|l|}{ Modelo completo } \\
\hline D*Sist. Prod. ${ }^{\square}$ & 9,58 & 26,86 & $-9,45$ & 27,43 & 2,30 & 22,83 \\
\hline $\mathrm{D}^{2} \mathrm{H}^{*}$ Sist. Prod. & 9,49 & 23,19 & $-8,36$ & 28,37 & 2,65 & 20,80 \\
\hline $\begin{array}{l}\text { D*Sist. Prod, H*Sist. } \\
\text { Prod }\end{array}$ & 10,67 & 27,55 & $-9,15$ & 28,00 & 3,16 & 23,54 \\
\hline
\end{tabular}

q = Sistema de producción. $\mathrm{D}$ = Diámetro al cuello de la raíz. $\mathrm{H}$ = Altura del tallo.

$\mathrm{SD}=$ Standar deviation . 
tal tuvieron tendencias similares; igualmente, Akpo et al. (2014) afirman que la estimación de la biomasa aérea lleva a conclusiones igualmente significativas que la estimación de la biomasa total. En cambio, para el cálculo de biomasa de la raíz, a pesar que el modelo completo presentó mejor ajuste, su error de predicción fue mayor en los datos de validación en comparación con el modelo reducido; estas discrepancias concuerdan con lo manifestado por Niklas (2004), quien señala que la estimación de la biomasa de la raíz depende progresivamente del tamaño de planta, debido a que las plantas más grandes tienen desproporcionadamente más raíces finas y en consecuencia son más difíciles de explorar.

Finalmente, tal como lo sugieren Ter-Mikaelian y Parker (2000) y Bloomberg et al. (2008) para otras especies de coníferas, el modelo se ajustó eficientemente en la predicción de biomasa en plantas de Pinus pseudostrobus y los resultados del análisis de covarianza indican que es ne-
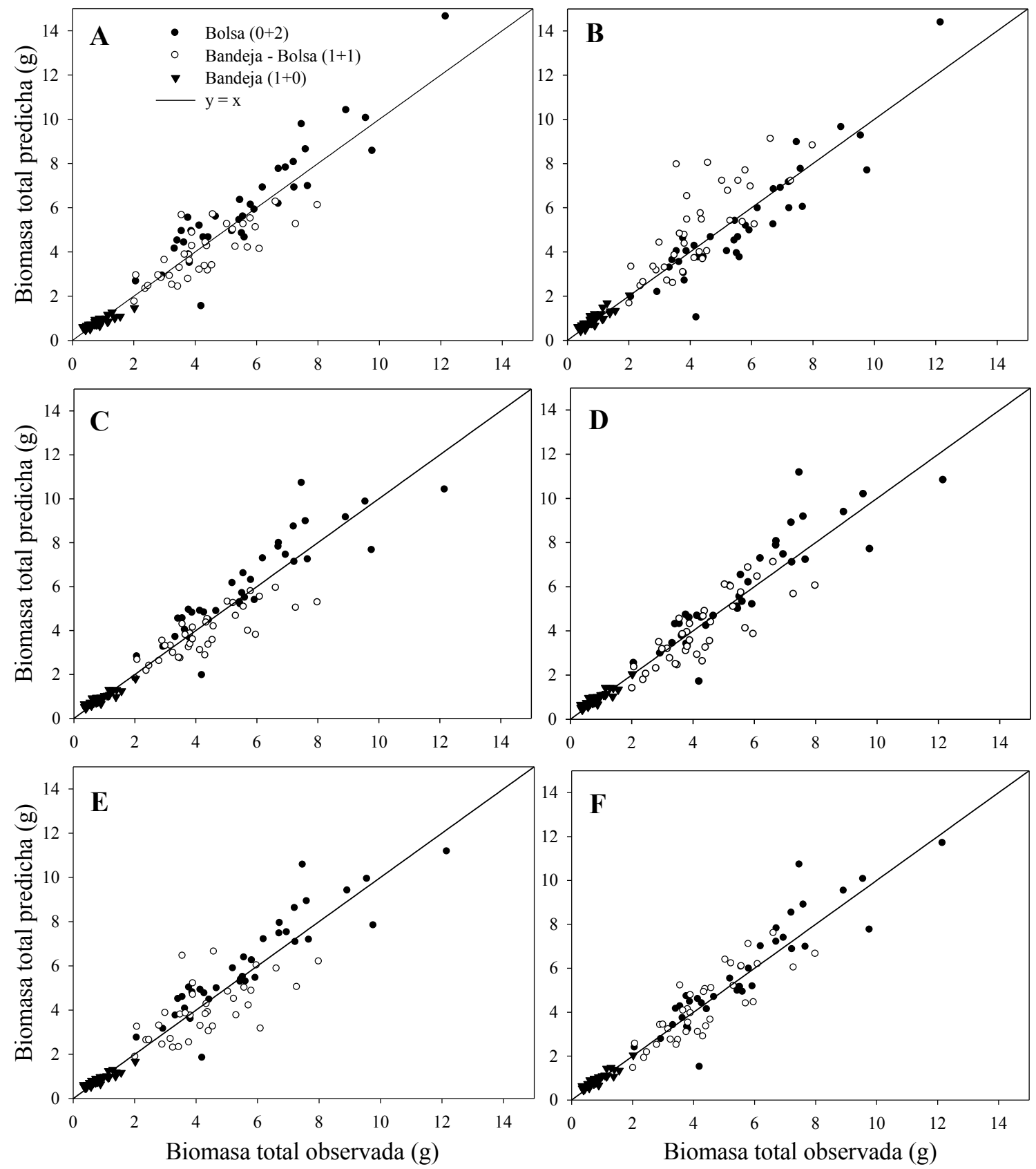

Figura 3. Biomasa total predicha contra biomasa total observada en los datos de validación $(\mathrm{n}=108)$. Modelo completo para $\mathrm{D}(\mathrm{A})$, $\mathrm{D}^{2} \mathrm{H}(\mathrm{C})$ y D, H (E) y modelo reducido para D (B), D ${ }^{2} \mathrm{H}(\mathrm{D})$ y D, H (F).

Predicted total biomass vs. observed total biomass in the validation dataset $(\mathrm{n}=108)$. Full model for $D(\mathrm{~A}), D^{2} H(\mathrm{C})$, and $D, H(\mathrm{E})$, and reduced model for $D(\mathrm{~B}), D^{2} H(\mathrm{D})$, and $D, H(\mathrm{~F})$. 
cesario utilizar una ecuación incluyendo el sistema de producción para mejorar las predicciones de la biomasa aérea $\mathrm{y}$ total. Esto permitirá reducir el número de plantas muestreadas alcanzando la misma precisión y minimizando el tiempo y los costos del muestreo (Climent et al. 2008). Por lo tanto, las ecuaciones generadas son una herramienta para el monitoreo de los índices de calidad de planta durante su desarrollo en vivero, sin recurrir a muestreos destructivos; igualmente, pueden ser utilizarlas para estimar la acumulación de biomasa en plantaciones a etapas tempranas después de su establecimiento.

\section{CONCLUSIONES}

El sistema de producción muestra efecto significativo sobre el intercepto y pendiente de las relaciones alométricas para predecir biomasa aérea y total, no así para la biomasa de la raíz.

En este estudio se demuestra que es posible estimar la biomasa total y aérea en plantas de Pinus pseudostrobus mediante ecuaciones alométricas específicas para cada sistema de producción en vivero, utilizando el diámetro al cuello de la raíz y altura del tallo como variables predictivas.

Para la estimación de la biomasa de la raíz, alcanza el mejor ajuste el modelo reducido que incluye sólo el diámetro como variable predictiva, sin considerar el sistema de producción. En este sentido, el diámetro al cuello de la raíz es la variable que mejor predice la variación de biomasa en cada componente; no obstante, al calcular la biomasa aérea, la altura del tallo debe ser considerada como un buen predictor, ya que es la característica más sensible al diferenciar condiciones de producción o manejo de la planta en vivero.

\section{REFERENCIAS}

Aguirre COA, J Jiménez P. 2011. Evaluación del contenido de carbono en bosques del sur de Nuevo León. Revista Mexicana de Ciencias Forestales 2(6): 73-83.

Akpo E, TJ Stomph, DK Kossou, AO Omore, PC Struik. 2014. Effects of nursery management practices on morphological quality attributes of tree seedlings at planting: The case of oil palm (Elaeis guineensis Jacq.). Forest Ecology and Management 324: 28-36.

Bloomberg M, EG Mason, P Jarvis, R Sedcole. 2008. Predicting seedling biomass of radiata pine from allometric variables. New Forests 36: 103-114.

Blujdea VNB, R Pilli, I Dutca, L Ciuvat, IV Abrudan. 2011. Allometric biomass equations for young broadleaved trees in plantations in Romania. Forest Ecology and Management 264: 172-184.

Chaturvedi RK, AS Raghubanshi. 2013. Aboveground biomass estimation of small diameter woody species of tropical dry forest. New Forests 44(4): 509-519.

Climent J, J Alonso, L Gil. 2008. Short note: root restriction hindered early allometric differentiation between seedlings of two provenances of Canary Island pine. Silvae Genetica 57: 4-5.

CONAFOR (Comisión Nacional Forestal, MX). 2011. Indicadores de gestión de desempeño del programa PROCOREF durante el ejercicio fiscal 2010. Universidad Autónoma Chapingo (UACH). Consultado 23 ene. 2013. Disponible en http://www.cnf.gob.mx:8080/snif/portal/evaluaciones/ documentos-y-alcances-de-las-evaluaciones

Del Campo AD, J Hermoso, CJ Ceacero, RM Navarro-Cerrillo. 2011. Nursery location and potassium enrichment in Aleppo pine stock 1. Effect on nursery culture, growth, allometry and seedling quality. Forestry 84(3): 221-234.

Dickson A, AL Leaf, JF Hosner. 1960. Seedling quality - soil fertility relationships of white spruce, red, and white pine in nurseries. The Forest Chronicle 36(3): 237-241.

Domínguez CG, OA Aguirre C, J Jiménez P, R Rodríguez L, JA Díaz B. 2009. Biomasa aérea y factores de expansión de especies arbóreas en bosques del sur de Nuevo León. Revista Chapingo. Serie Ciencias Forestales y del Ambiente 15(1): 59-64

Geudens G, J Staelens, V Kint, R Goris, N Lust. 2004. Allometric biomass equations for Scots pine (Pinus sylvestris L.) seedlings during the first years of establishment in dense natural regeneration. Annals of Forest Sciences 61: 653-659.

Grossnickle SC. 2012. Why seedlings survive: influence of plant attributes. New Forests 43: 711-738.

Jackson DP, RK Dumroese, JP Barnett. 2012. Nursery response of container Pinus palustris seedlings to nitrogen supply and subsequent effects on outplanting performance. Forest Ecology and Management 265: 1-12.

Lödige C, P Schall, C Ammer. 2014. How do size and resource availability control aboveground biomass allocation of tree seedlings? Forest Research 3: 123.

Méndez GJ, SL Luckie N, MA Capó A, JANájera. 2011. Ecuaciones alométricas y estimación de incrementos en biomasa aérea y carbono en una plantación mixta de Pinus devoniana lindl. y P. pseudostrobus lindl., en Guanajuato, México. Agrociencia 45: 479-491.

Miguel C, X Aranda, F De Herralde, S Sabaté, C Biel, R Savé. 2010. Evaluation of growth slowdown nursery treatments on Prunus avium seedlings by means of allometric relationships and relative growth rates. Scandinavian Journal of Forest Research 25(S8): 51-59.

Niklas KJ. 2004. Plant allometry: is there a grand unifying theory? Biological Reviews 79(4): 871-889.

Ortega U, J Majada, A Mena P, J Sánchez Z, N Rodríguez I, K Txarterina, J Azpitarte, M Duñabeitia. 2006. Field performance of Pinus radiata D. Don produced in nursery with different types of containers. New Forests 31: 97-112.

Preece ND, MJ Lawes, AK Rossman, TJ Curran, P van Oosterzee. 2015. Modelling the growth of young rainforest trees for biomass estimates and carbon sequestration accounting. Forest Ecology and Management 351(1): 28-36.

Ruehle JL, DH Marx, HD Muse. 1984. Calculated nondestructive indices of growth response for young pine seedlings. Forest Science 30(2): 469-474.

SAS (Statistical Analysis System Institute Inc.). 2009. SAS Ver. 9.2. SAS Inc., Cary, NC.

Schmidt A, M Poulain, D Klein, K Krause, K Peña-Rojas, H Schmidt, A Schulte. 2009. Allometric above-belowground biomass equations for Nothofagus pumilio (Poepp. et Endl.) natural regeneration in the Chilean Patagonia. Annals of 
Forest Sciences 66: 513.

Sprugel DG. 1983. Correcting for bias in Log-transformation allometric equations. Ecology 64(1): 209-210.

Stape JL, JL Moraes G, A Natal G. 2001. Relationships between nursery practices and field performance for Eucalyptus plantations in Brazil. New Forests 22: 19-41.

Takahashi K, Y Obata. 2014. Growth, allometry and shade tolerance of understory saplings of four subalpine conifers in central Japan. Journal of Plant Research 127(2): 329-338.
Tsakaldimi M, P Ganatsas, DF Jacobs. 2013. Prediction of planted seedlings survive of five Mediterranean species based on initial seedling morphology. New Forests 44: 327-339.

Ter-Mikaelian M, M Korzukhin. 1997. Biomass equations for sixty-five North American tree species. Forest Ecology and Management 97: 1-24.

Ter-Mikaelian MT, WC Parker. 2000. Estimating biomass of white spruce seedlings with vertical photo imagery. New Forests 20: 145-162.

Recibido: 22.09 .15

Aceptado: 11.04.16 